LEADING ARTICLE

\title{
Colorectal cancer prognosis: is it all mutation, mutation, mutation?
}

A B Hassan, C Paraskeva

For the 500000 new cases of colorectal cancer in the world each year, identification of patients with a worse prognosis and those who are more likely to respond to treatment is a challenge. There is an increasing body of evidence correlating genetic mutations with outcome in tumours derived from human colorectal cancer cohorts. K-ras, but not p53 or APC, mutations appear to be associated with poorer overall survival in colorectal cancer patients.

See end of article for authors' affiliations

.....................

Correspondence to:

Professor A B Hassan,

Department of Cellular and

Molecular Medicine

(formerly Pathology and

Microbiology), School of

Medical Sciences,

University of Bristol, Bristol

BS8 1TD, UK;

Bass.Hassan@

Bristol.ac.uk

Revised version received 24 May 2005

Accepted for publication

26 May 2005 the 1920s. While there is nothing immediately wrong with this classification and its derivatives, in particular because it has been universally adopted, it is clear that significant heterogeneity exists between patient outcomes with otherwise apparently identical pathological staging. The immediate challenge faced by the clinical community is to identify patients with a worse prognosis and those who are more likely to respond to treatments, such as surgical resection, radiotherapy, chemotherapy, and molecular therapy.

Patient selection is also now more of an important issue for a series of scientifically based reasons. These include identification of patients at high risk from colorectal cancer prior to screening and prevention programmes, those with familial predisposition (35\% of total), including mutation screening of key genes (2$6 \%$ of total), and more recently in systemic treatment, as empirically applied treatments including cytotoxic chemotherapy are now being challenged by new molecular therapies. Some of the new molecules are currently administered empirically, as the co-development of molecular testing and tailored treatments has not kept pace with the enthusiasm of the immediate therapeutic testing. ${ }^{12}$ In time, this situation will reverse as the costs of treatments increase, and we should expect a series of predictive tests to be developed that facilitate selection based on currently available targeted therapy. In situations where targeted molecular therapy has been developed example, breast (Her2), chronic myeloid leukaemia (BCR-ABL), gastrointestinal stromal
Gut 2005;54:1209-1211. doi: 10.1136/gut.2005.070946

there have been dramatic improvements in disease management that far exceed anything that has been achieved with an empirical approach. What is critical is that the selectivity and potency of these therapies exploits mutation in the tumour cells, with amplification of Her2, translocation in BCR-ABL, and mutation of receptors C-Kit and EGFR. Although an expensive challenge, the cancer field is rightly continuing to pursue the identification and evaluation of common human mutations in cancer, as evident by the Cancer Genome project. ${ }^{3}$ This activity will no doubt drive the development of specific therapeutic interventions to specific mutated targets. Despite the success to date in other cancers, this whole activity could be an expensive mistake if a combination of mutation complexity, aneuploidy, and epigenetic alterations in tumour cells ultimately determine prognosis and response to therapy, irrespective of targeted therapy to what are presumed to be key mutations. with a validated molecular diagnostic test (for tumours (C-KIT), and lung cancer (EGFR)),

\begin{abstract}
"Although an expensive challenge, the cancer field is rightly continuing to pursue the identification and evaluation of common human mutations in cancer, as evident by the Cancer Genome project"
\end{abstract}

So what of mutations in colorectal cancer? The paper by the Dundee group ${ }^{4}$ in this issue of Gut contributes to an increasing body of evidence correlating mutation with outcome in tumours derived from human colorectal cancer cohorts (see page 1283). Before examining their findings, it is important to recognise that mutational analysis and genetic models have already identified the key pathways critical for initiation and progression of colorectal cancer. Deregulation of the Wnt signalling pathway, by either loss of function or gain of function mutations of adenomatous polyposis coli (APC) and $\beta$-catenin, respectively, in both hereditary and sporadic colorectal adenoma, establish the principal initiation pathway. ${ }^{5}$ Although specific mutational hotspots occur that account for attenuated inherited phenotypes, most sporadic mutations of APC occur in a mutational cluster. ${ }^{67}$ The discriminating power and clinical utility of these mutations have proven to be less impressive, even though this pathway remains a critical therapeutic target. ${ }^{8}$ Genetic modifiers of the number, differentiation, and progression of Abbreviations: K-ras, Kirsten-ras; APC, adenomatous
polyposis coli 
colorectal adenoma can either derive from the host or from coexisting and selected mutations in the tumour. Dissecting these components has been problematic, mainly because a large number of variables exist (for example, variable penetrance, gain and loss of function mutations in each gene, and synergistic effects between modifiers that coexist in both tumour and stromal cell populations). Although these limitations are being circumvented using larger patient numbers with high throughput technologies, there will have to be a practical outcome that will essentially distil down this information to a fewer number of key mutations, and potentially to where we have started from, a classification based on morphology and identification of a subpopulation of localised cells that essentially determine overall outcome (for example, those at the micro-invasive front of the tumour).

"K-ras mutations, but not p53 or APC mutations, were associated with poorer overall survival, even when correcting for Dukes' stage, age, and sex"

The Dundee group prospectively characterised mutations in three genes implicated in colorectal cancer $(A P C, p 53$, Kirstenras $(K$-ras $))$ and extended a previous study. ${ }^{9}$ They observed that the mutation frequencies were variable between genes $(A P C=56 \%, p 53=61 \%, K$-ras $=27 \%)$, and when they controlled for expected frequencies, p53 and K-ras genes rarely coexisted (observed $2 \%$ versus expected $16 \%$ ), even though the reduction in expected frequency of combined p53 and K-ras appears to have been lost when mutations of APC coexisted in addition. Conlin and colleagues ${ }^{4}$ have also now correlated these mutations with survival in colorectal cancer. In a relatively small cohort of 107 patients, the significant additional findings were that K-ras mutations, but not p53 and APC mutations, were associated with poorer overall survival, even when correcting for Dukes' stage, age, and sex. The surprise is that the magnitude of this effect for a single gene appears large in this cohort relative to the influence of Dukes' stage alone, but on closer analysis, the frequency of mutations of K-ras was highest overall in more advanced disease. A similar examination of K-ras mutations in a larger cohort also showed association of the glycine codon 12 to valine mutation with high risk disease. ${ }^{10}$ Moreover, similar studies examining p53, chromosomal loss, and microsatellite instability also correlate with survival, with a subgroup recognised that neither falls within a chromosomal nor microsatellite unstable groups. ${ }^{11}{ }^{12}$

How should we view this information? Clearly, some of the data concern small cohorts, and the magnitude of the effects may be lost in larger studies. Moreover, the complexity of this analysis is complicated because mutations in some genes do not always generate the same phenotypic outcomes. For example, for $\mathrm{p} 53$, recent data implicate specific mutations of p53 with colorectal cancer outcome, although there does not appear to be an overall correlation, as Conlin et al have suggested. ${ }^{13-15}$ Ultimately, mutations and chromosomal abnormalities that are both easier to detect and at high frequencies will be selected first, simply because of the eventual contribution to statistical outcome. ${ }^{16}{ }^{17}$ Some would also argue that a focus solely on mutation would distract from the potential contribution of epigenetic modification of modifier gene expression, which we know is also commonly detected in colorectal cancer.

"Ultimately, mutations and chromosomal abnormalities that are both easier to detect and at high frequencies will be selected first, simply because of the eventual contribution to statistical outcome"
So, are we making too many assumptions about the complexity of the task ahead? Probably not when it comes to deriving useful information, because studies such as Conlin et al highlight this fact in terms of specific and common gene mutational frequency in colorectal cancer tissue derived from patients. In addition, the few data there are, are also being immediately assembled with Dukes' stage information, site, and ploidy into an increasing number of subgroups, despite the relatively small cohorts examined. ${ }^{18}$ The complexity is very high and no one should underestimate the difficulty. Not surprisingly, there is a need for newer approaches, perhaps to assemble mutations into various pathways, which are best exemplified by the six hallmarks proposed by Hanahan and Weinberg, ${ }^{19}$ and to then analyse what intermediate surrogate markers may be used to subdivide and target mutation testing. This may not mean an initial screen for all the potential mutually exclusive mutations along one pathway, but a "readout" of its overall activity based on histological, proteomic, or expression profiling..$^{20-22}$ The goal of the latter must be to ultimately apply specific interventions, and to test the usefulness of the mutation in the overall context of prevention, diagnosis, prognosis, and therapy. In this case, mutations, and epigenetic and expression alterations are then judged by clinical utility.

\section{ACKNOWLEDGEMENTS}

We acknowledge research funding support from Cancer Research UK.

\section{Authors' affiliations}

A B Hassan, C Paraskeva, Department of Cellular and Molecular Medicine (formerly Pathology and Microbiology), School of Medical Sciences, University of Bristol, UK

Conflict of interest: None declared.

\section{REFERENCES}

1 Cunningham D, Humblet $Y$, Siena S, et al. Cetuximab monotherapy and cetuximab plus irinotecan in irinotecan-refractory metastatic colorectal cancer. N Engl J Med 2004;351:337-45.

2 Hurwitz H, Fehrenbacher L, Novotny W, et al. Bevacizumab plus irinotecan, fluorouracil, and leucovorin for metastatic colorectal cancer. N Engl J Med 2004;350:2335-42

3 Gabor Miklos GL. The Human Cancer Genome Project-one more misstep in the war on cancer. Nat Biotechnol 2005;23:535-7.

4 Conlin A, Smith G, Carey FA, et al. The prognostic significance of K-ras, p53, and APC mutations in colorectal carcinoma. Gut 2005;54:1283-6.

5 Reya T, Clevers H. Wht signalling in stem cells and cancer. Nature 2005;434:843-50.

6 Crabtree MD, Tomlinson IP, Hodgson SV, et al. Explaining variation in familial adenomatous polyposis: relationship between genotype and phenotype and evidence for modifier genes. Gut 2002;51:420-3.

7 de la Chapelle A. Genetic predisposition to colorectal cancer. Nat Rev Cancer 2004;4:769-80.

8 Lepourcelet $M$, Chen YN, France DS, et al. Small-molecule antagonists of the oncogenic Tcf/beta-catenin protein complex. Cancer Cell 2004;5:91-102.

9 Smith G, Carey FA, Beattie J, et al. Mutations in APC, Kirsten-ras, and p53alternative genetic pathways to colorectal cancer. Proc Natl Acad Sci U S A 2002;99:9433-8.

10 Andreyev HJ, Norman AR, Cunningham D, et al. Kirsten ras mutations in patients with colorectal cancer: the 'RASCAL II' study. Br J Cancer 2001;85:692-6.

11 Hawkins NJ, Tomlinson I, Meagher A, et al. Microsatellite-stable diploid carcinoma: a biologically distinct and aggressive subset of sporadic colorectal cancer. Br J Cancer 2001;84:232-6.

12 Georgiades IB, Curtis $\sqcup$, Morris RM, et al. Heterogeneity studies identify a subset of sporadic colorectal cancers without evidence for chromosomal or microsatellite instability. Oncogene 1999;18:7933-40.

13 Diep CB, Thorstensen L, Meling Gl, et al. Genetic tumor markers with prognostic impact in Dukes' stages B and C colorectal cancer patients. J Clin Oncol 2003;21:820-9.

14 Russo A, Migliavacca M, Zanna l, et al. p53 mutations in L3-loop zinc-binding domain, DNA-ploidy, and S phase fraction are independent prognostic indicators in colorectal cancer: a prospective study with a five-year follow-up. Cancer Epidemiol Biomarkers Prev 2002;11:1322-31. 
15 Forslund A, Lonnroth C, Andersson M, et al. Mutations and allelic loss of p53 in primary tumor DNA from potentially cured patients with colorectal carcinoma. J Clin Oncol 2001;19:2829-36.

16 Sieber OM, Heinimann K, Tomlinson IP. Genomic instability-the engine of tumorigenesis? Nat Rev Cancer 2003;3:701-8.

17 Vendrell E, Morales C, Risques RA, et al. Genomic determinants of prognosis in colorectal cancer. Cancer Lett 2005;221:1-9.

18 Risques RA, Moreno V, Ribas M, et al. Genetic pathways and genome-wide determinants of clinical outcome in colorectal cancer. Cancer Res 2003:63:7206-14.

19 Hanahan D, Weinberg RA. The hallmarks of cancer. Cell 2000;100:57-70.
20 Nishizuka S, Charboneau L, Young L, et al. Proteomic profiling of the $\mathrm{NCl}-60$ cancer cell lines using new high-density reverse-phase lysate microarrays. Proc Natl Acad Sci U S A 2003; 100:14229-34.

21 Nishizuka S, Chen ST, Gwadry FG, et al. Diagnostic markers that distinguish colon and ovarian adenocarcinomas: identification by genomic, proteomic, and tissue array profiling. Cancer Res 2003;63:5243-50.

22 Kimura ET, Nikiforova MN, Zhu Z, et al. High prevalence of BRAF mutations in thyroid cancer: genetic evidence for constitutive activation of the RET/PTCRAS-BRAF signaling pathway in papillary thyroid carcinoma. Cancer Res 2003;63:1454-7

\section{Clinical Evidence - Call for contributors}

Clinical Evidence is a regularly updated evidence-based journal available worldwide both as a paper version and on the internet. Clinical Evidence needs to recruit a number of new contributors. Contributors are healthcare professionals or epidemiologists with experience in evidence-based medicine and the ability to write in a concise and structured way.

Areas for which we are currently seeking authors:

- Child health: nocturnal enuresis

- Eye disorders: bacterial conjunctivitis

- Male health: prostate cancer (metastatic)

- Women's health: pre-menstrual syndrome; pyelonephritis in non-pregnant women However, we are always looking for others, so do not let this list discourage you.

Being a contributor involves:

- Selecting from a validated, screened search (performed by in-house Information Specialists) epidemiologically sound studies for inclusion.

- Documenting your decisions about which studies to include on an inclusion and exclusion form, which we keep on file.

- Writing the text to a highly structured template (about 1500-3000 words), using evidence from the final studies chosen, within 8-10 weeks of receiving the literature search.

- Working with Clinical Evidence editors to ensure that the final text meets epidemiological and style standards.

- Updating the text every six months using any new, sound evidence that becomes available. The Clinical Evidence in-house team will conduct the searches for contributors; your task is simply to filter out high quality studies and incorporate them in the existing text.

- To expand the topic to include a new question about once every 12-18 months.

If you would like to become a contributor for Clinical Evidence or require more information about what this involves please send your contact details and a copy of your CV, clearly stating the clinical area you are interested in, to Klara Brunnhuber (kbrunnhuber@ bmigroup.com).

\section{Call for peer reviewers}

Clinical Evidence also needs to recruit a number of new peer reviewers specifically with an interest in the clinical areas stated above, and also others related to general practice. Peer reviewers are healthcare professionals or epidemiologists with experience in evidence-based medicine. As a peer reviewer you would be asked for your views on the clinical relevance, validity, and accessibility of specific topics within the journal, and their usefulness to the intended audience (international generalists and healthcare professionals, possibly with limited statistical knowledge). Topics are usually 1500-3000 words in length and we would ask you to review between 2-5 topics per year. The peer review process takes place throughout the year, and our turnaround time for each review is ideally 10-14 days.

If you are interested in becoming a peer reviewer for Clinical Evidence, please complete the peer review questionnaire at www.clinicalevidence.com or contact Klara Brunnhuber (kbrunnhuber@bmigroup.com). 